\title{
Versatile click alginate hydrogels crosslinked via tetrazine-norbornene chemistry
}

\section{Citation}

Desai, Rajiv M., Sandeep T. Koshy, Scott A. Hilderbrand, David J. Mooney, and Neel S. Joshi. 2015. "Versatile Click Alginate Hydrogels Crosslinked via Tetrazine-norbornene Chemistry." Biomaterials 50 (May): 30-37. doi:10.1016/j.biomaterials.2015.01.048.

\section{Published Version}

doi:10.1016/j.biomaterials.2015.01.048

\section{Permanent link}

http://nrs.harvard.edu/urn-3:HUL.InstRepos:14531727

\section{Terms of Use}

This article was downloaded from Harvard University's DASH repository, and is made available under the terms and conditions applicable to Open Access Policy Articles, as set forth at http:// nrs.harvard.edu/urn-3:HUL.InstRepos:dash.current.terms-of-use\#OAP

\section{Share Your Story}

The Harvard community has made this article openly available.

Please share how this access benefits you. Submit a story.

Accessibility 
Title: Versatile click alginate hydrogels crosslinked via tetrazine-norbornene chemistry

Authors: Rajiv Desai ${ }^{1,2, \star}$, Sandeep T. Koshy ${ }^{1-3, \star}$, Scott A. Hilderbrand ${ }^{4,5}$, David J. Mooney ${ }^{1,2, \S}$, Neel S. Joshi ${ }^{1,2, \S}$

\section{Affiliations:}

${ }^{1}$ School of Engineering and Applied Sciences, Harvard University, Cambridge, MA 02138, USA.

${ }^{2}$ Wyss Institute for Biologically Inspired Engineering, Harvard University, Boston, MA 02115, USA.

${ }^{3}$ Harvard-MIT Division of Health Sciences and Technology, Cambridge, MA 02139, USA.

${ }^{4}$ Center for Systems Biology, Massachusetts General Hospital, Boston, MA 02114, USA.

${ }^{5}$ Harvard Medical School, Boston, MA 02114, USA

$\S$ To whom correspondence should be addressed. E-mail: mooneyd@seas.harvard.edu or njoshi@seas.harvard.edu

* These authors contributed equally to this work 


\section{Abstract:}

Alginate hydrogels are well-characterized, biologically inert materials that are used in many biomedical applications for the delivery of drugs, proteins, and cells. Unfortunately, canonical covalently crosslinked alginate hydrogels are formed using chemical strategies that can be biologically harmful due to their lack of chemoselectivity. In this work we introduce tetrazine and norbornene groups to alginate polymer chains and subsequently form covalently crosslinked click alginate hydrogels capable of encapsulating cells without damaging them. The rapid, bioorthogonal, and specific click reaction is irreversible and allows for easy incorporation of cells with high post-encapsulation viability. The swelling and mechanical properties of the click alginate hydrogel can be tuned via the total polymer concentration and the stoichiometric ratio of the complementary click functional groups. The click alginate hydrogel can be modified after gelation to display cell adhesion peptides for 2D cell culture using thiol-ene chemistry. Furthermore, click alginate hydrogels are minimally inflammatory, maintain structural integrity over several months, and reject cell infiltration when injected subcutaneously in mice. Click alginate hydrogels combine the numerous benefits of alginate hydrogels with powerful bioorthogonal click chemistry for use in tissue engineering applications involving the stable encapsulation or delivery of cells or bioactive molecules.

Keywords: Alginate, Hydrogel, Click chemistry, Cell adhesion, Cell encapsulation, Tissue engineering 


\section{Introduction:}

Hydrogels are highly hydrated, crosslinked polymer networks that resemble the environment of natural soft tissue, making them attractive materials for a variety of biomedical applications such as tissue engineering, drug delivery, and vaccines [1-7]. Alginate biopolymers are versatile, naturally derived linear polysaccharides comprised of repeating $(1,4)$-linked $\beta$-D-mannuronic and $\alpha$-Lguluronic acid, and can be crosslinked to form hydrogels via a variety of ionic and covalent crosslinking methods [8,9]. Alginate hydrogels can be engineered to release small molecules and proteins, present bioactive ligands to cells, and degrade at a tunable rate [10-12]. Furthermore, ionically crosslinked alginates have been used extensively for drug delivery, cell encapsulation, and tissue engineering because ionic crosslinking can be largely benign to cells and encapsulated molecules [13].

The encapsulation of various small molecules, proteins, and cells in alginate hydrogels has thus far been largely limited to the reversible ionic crosslinking method which uses divalent cations, such as $\mathrm{Ca}^{2+}$, to form ionic bridges between adjacent polymer chains. These gels have been shown to be weak and to lose mechanical integrity over time in vitro and in vivo due to the reversible nature of the crosslinking and subsequent outward flux of ions from the hydrogel [14]. Calcium crosslinked alginate gels can yield non-uniform physical properties, due to extremely rapid crosslinking with certain ions [15]. Moreover, leached calcium from calcium crosslinked alginate gels can be immunostimulatory, which is 
unfavorable in many in vivo applications [16]. While alginate is well characterized in its ability to quantitatively couple small molecules, peptides, and proteins to the polymer backbone, these reactions (e.g. carbodiimide couplings) are typically limited in efficiency by slow reaction kinetics under aqueous conditions [17].

To overcome many of the challenges associated with ionic crosslinking, alternative covalent crosslinking strategies have been developed, though none are completely biologically inert [18-21]. Many of these covalent crosslinking strategies produce stable and uniform gels with mechanical properties that are controllable over a wider range compared to ionically crosslinked gels, but they may not be optimal for protein or cell encapsulation due to the cross-reactivity of the crosslinking chemistry with cells and proteins. Additionally, as the quantity and length of the crosslinker increases, the properties of the resulting hydrogel are significantly altered, making it difficult to compare such gels to alginate-based ionically crosslinked hydrogels [22].

Click chemistry has recently emerged as an alternative approach to synthesize covalently crosslinked hydrogels with high chemoselectivity and fast reaction rates in complex aqueous media, at physiologically relevant $\mathrm{pH}$ and temperature ranges both in vitro and in vivo [23]. Recent findings have established a set of bioorthogonal click reactions that do not require the cytotoxic copper catalyst used in early reports. These copper-free chemistries include strain-promoted azide-alkyne cycloaddition (SPAAC) and the inverse electron demand Diels-Alder reaction between tetrazine and norbornene [24,25]. Previous 
reports have used these click reactions primarily to crosslink click endfunctionalized branched polyethylene glycol (PEG) with linear crosslinkers composed of either PEG or linear peptides terminated with the appropriate click reaction pair [26-29]. The mechanical properties and swelling behavior of these click crosslinked PEG hydrogels could be tuned by varying the linear crosslinker concentration $[30,31]$.

We hypothesized that a simpler and more robust click crosslinked biomaterial could be designed to exhibit stable and tunable mechanical properties, present bioactive ligands to cells, and encapsulate those cells in a cytocompatible covalent crosslinked alginate hydrogel. In this report, we modified alginate biopolymers with tetrazine or norbornene functional groups, allowing for covalent crosslinking without the need for external input of energy, crosslinkers, or catalysts, using the bioorthogonal inverse electron demand Diels-Alder click reaction. In addition to the crosslinking reaction, the click alginate system exploits photoinitated thiol-ene based modification of the norbornene groups to present thiol-bearing peptides or fluorescent dyes. We investigated cell adhesion on the hydrogel surface and cell growth and viability when encapsulated in 3D in click alginate hydrogels. In addition, we studied the host inflammatory response to click alginate hydrogels that are injected in vivo.

\section{Materials and Methods:}

2.1 3-(p-benzylamino)-1,2,4,5 tetrazine synthesis 
3-(p-benzylamino)-1,2,4,5-tetrazine was synthesized according to an established protocol [32]. Briefly, $50 \mathrm{mmol}$ of 4-(aminomethyl)benzonitrile hydrochloride and $150 \mathrm{mmol}$ formamidine acetate were mixed while adding $1 \mathrm{~mol}$ of anhydrous hydrazine. The reaction was stirred at $80{ }^{\circ} \mathrm{C}$ for 45 minutes and then cooled to room temperature, followed by addition of $0.5 \mathrm{~mol}$ of sodium nitrite in water. $10 \% \mathrm{HCl}$ was then added dropwise to acidify the reaction to form the desired product. The oxidized acidic crude mixture was then extracted with DCM. After discarding the organic fractions, the aqueous layer was basified with $\mathrm{NaHCO}_{3}$, and immediately extracted again with $\mathrm{DCM}$. The final product was then recovered by rotary evaporation, and purified by HPLC. All chemicals were purchased from Sigma-Aldrich.

\subsection{Click alginate polymer synthesis}

Click alginate biopolymers were modified with either 1-bicyclo[2.2.1]hept-5en-2-ylmethanamine (Norbornene Methanamine; Matrix Scientific) or 3-( $p$ benzylamino)-1,2,4,5-tetrazine by first allowing high molecular weight alginate, $M_{w}=265 \mathrm{kDa}$ (Protanol LF 20/40; FMC Technologies) to dissolve in stirred buffer containing $0.1 \mathrm{M} \mathrm{MES}, 0.3 \mathrm{M} \mathrm{NaCl}, \mathrm{pH} 6.5$ at $0.5 \% \mathrm{w} / \mathrm{v}$. Next, $N$ hydroxysuccinimide (NHS; Sigma-Aldrich) and 1-ethyl-3-(3dimethylaminopropyl)-carbodiimide hydrochloride (EDC; Sigma-Aldrich) were added in 5x molar excess of the carboxylic acid groups of alginate. Either norbornene or tetrazine was then added at $1 \mathrm{mmol}$ per gram of alginate to make 
Alg-N or Alg-T, respectively. The coupling reaction was stirred at room temperature for 24 hours, after which the reaction was quenched with hydroxylamine (Sigma-Aldrich) and dialyzed in 12-14 kDa MWCO dialysis tubing (Spectrum Labs) for 4 days against a decreasing salt gradient from $150 \mathrm{mM}$ to 0 $\mathrm{mM} \mathrm{NaCl}$ in $\mathrm{diH}_{2} \mathrm{O}$. The purified Alg-N and Alg-T polymers were treated with activated charcoal, sterile filtered $(0.22 \mu \mathrm{m})$, and freeze-dried. This resulted in purified Alg-N or Alg-T polymers with a $5 \%$ degree of substitution of the available carboxylic acid groups of alginate. (Fig. S-1).

\subsection{Preparation and characterization of click alginate hydrogels}

Click alginate hydrogels were prepared by first separately dissolving freezedried Alg- $\mathrm{N}$ and Alg-T polymers to final desired concentration (2-4\% w/v) in Dulbecco's Modified Eagle Medium (DMEM; Gibco). For gelation kinetics measurements, Alg-N and Alg-T polymer solutions were mixed at a desired ratio (i.e., $0.5-4: 1 \mathrm{~N}: \mathrm{T}$ ) and directly pipetted onto the bottom plate of a TA Instruments ARG2 rheometer equipped with $8 \mathrm{~mm}$ flat upper plate geometry. A Peltier cooler was used to control the temperature for temperature dependent experiments, and mineral oil was applied to the gel periphery to prevent the hydrogel from drying during testing. Hydrogel samples were subjected to $1 \%$ strain at $1 \mathrm{~Hz}$, and the storage and loss moduli (G' and G") were monitored for 4 hours. For Young's modulus measurements click alginate hydrogels were formed under siliconized glass plates (Sigmacote; Sigma-Aldrich) with $2 \mathrm{~mm}$ spacers. After 2 hours of 
crosslinking at room temperature, cylindrical disks were punched using an $8 \mathrm{~mm}$ biopsy punch, transferred to DMEM, and swollen to equilibrium for 24 hours at 37 ${ }^{\circ} \mathrm{C}$. Swollen hydrogel sample dimensions were measured using calipers for volumetric swelling ratio measurements, and then subjected to unconfined compression testing $(1 \mathrm{~mm} / \mathrm{min})$ using a $10 \mathrm{~N}$ load cell with no preload (Instron Model 3342). The Young's modulus, E, was calculated as the slope of the linear portion (first 10\%) of the stress vs. strain curves.

\subsection{Post-gelation thiol-ene photoreaction onto click alginate hydrogels}

Click alginate hydrogels were made as previously described $(2 \% \mathrm{w} / \mathrm{v}, \mathrm{N}: \mathrm{T}=$ 2) and then a cell adhesive CGGGGRGDSP peptide (Peptide2.0) solution at 0.2 or $2 \mathrm{mM}$ containing $0.5 \% \mathrm{w} / \mathrm{v}$ photoinitiator (Irgacure 2959; Sigma-Aldrich) was pipetted on top and the gel was covered with a glass coverslip. Gels were irradiated at $365 \mathrm{~nm}$ for 60 seconds at $10 \mathrm{~mW} / \mathrm{cm}^{2}$. The gels were washed several times with DMEM to remove excess photoinitiator and unreacted peptide and swollen to equilibrium at $37^{\circ} \mathrm{C}$ before seeding with cells.

\subsection{EGFP 3T3 cell culture}

NIH 3T3 (ATCC) cells were transduced with lentivirus produced from an EGFP-containing lentiviral vector (pLCAG EGFP, Inder Verma lab, Addgene plasmid 14857) [33] and were selected for 7 days in $1 \mu \mathrm{g} / \mathrm{mL}$ puromycin

dihydrochloride (EMD Millipore). EGFP-expressing 3T3 fibroblast cells were 
cultured in DMEM supplemented with $10 \%(\mathrm{v} / \mathrm{v})$ fetal calf serum, $100 \mathrm{U} / \mathrm{mL}$ penicillin, and $100 \mu \mathrm{g} / \mathrm{mL}$ streptomycin (Gibco) at $37^{\circ} \mathrm{C}$, in a $5 \% \mathrm{CO}_{2}$ environment. Cells were passaged approximately twice per week.

\subsection{Cell adhesion}

For cell adhesion studies, slabs of click alginate hydrogels were modified with cell adhesion peptides as described above. $6 \mathrm{~mm}$ disks were punched, placed in DMEM, washed several times, and swollen for 4 hours prior to seeding with cells at $5 \times 10^{4}$ cells $/ \mathrm{mL}$ at a depth of approximately $1 \mathrm{~mm}$ above the surface of the gel. Cells were given 24 hours to adhere and spread and then visualized via EGFP fluorescence using an epifluorescence microscope. EGFP images were used to quantify total cell area using ImageJ software. After 3 days of culture, cells were fixed and stained using Alexa Fluor 594 phalloidin (Molecular Probes) and Hoescht 33342 (Molecular Probes) to visualize F-actin filaments and nuclei respectively. To visualize cell death, gels were incubated for 20 minutes with a 4 $\mu \mathrm{M}$ ethidium homodimer-1 (Molecular Probes) solution in Hanks Buffered Saline Solution (HBSS) and imaged using an epifluorescence microscope.

\subsection{Cell encapsulation}

For cell encapsulation studies, Alg-N polymers were modified to have approximately 20 cell adhesive GGGGRGDSP peptides (Peptide2.0) per alginate chain as previously described [17]. $600 \mu \mathrm{m}$ thick click alginate hydrogels at $2 \%$ 
$\mathrm{w} / \mathrm{v}, \mathrm{N}: \mathrm{T}=1$, were then made containing cells at $3 \times 10^{6} \mathrm{cells} / \mathrm{mL}$. Ionically crosslinked hydrogels were similarly prepared at $2 \% \mathrm{w} / \mathrm{v}$ using the same cell density and backbone RGD modified Alg-N polymers. $\mathrm{A} \mathrm{CaSO}_{4}$ slurry $(0.21 \mathrm{~g}$ $\mathrm{CaSO}_{4} / \mathrm{mL} \mathrm{ddH} \mathrm{m}_{2} \mathrm{O}$ ) at a final concentration of $2 \% \mathrm{w} / \mathrm{v}$ was used to crosslink the ionically crosslinked hydrogel samples so as to match the mechanical properties of the two substrates as closely as possible. To minimize the time in which cells did not have access to culture media, gels were allowed to crosslink at room temperature for 1 hour, after which $6 \mathrm{~mm}$ disks were punched and placed in culture medium where the crosslinking reaction was expected to proceed to completion.

\section{$2.83 D$ in vitro cell assays}

Cells were retrieved from alginate hydrogels by digestion in a $5 \mathrm{U} / \mathrm{mL}$ alginate lyase (Sigma-Aldrich) solution in HBSS for 20 minutes. For viability testing, cells were stained with a Muse Count and Viability Kit and tested on a Muse Cell Analyzer (EMD Millipore). To assess total cell metabolic activity, gels were transferred to wells containing $10 \%$ AlamarBlue (AbD Serotec) in cell culture medium and incubated for 4 hours. The reduction of AlamarBlue was assessed according to the manufacturer's instructions.

\subsection{Mice}


All work was done with BALB/cJ mice (female, aged 6-8 weeks; Jackson Laboratories) and was performed in compliance with National Institutes of Health and institutional guidelines.

\subsection{In vivo hydrogel inflammatory response}

Ultrapure alginate with low endotoxin levels (MVG alginate, ProNova Biomedical AS) was modified as described above with norbornene and tetrazine and subsequently prepared at $2 \% \mathrm{w} / \mathrm{v}$ in DMEM after purification. Click alginate hydrogels were prepared by mixing ultrapure Alg-N and Alg-T polymers with $\mathrm{N}: \mathrm{T}$ $=1$ by connecting two syringes with a luer lock. 15 minutes after mixing, $50 \mathrm{uL}$ of click alginate hydrogel was injected subcutaneously through an $18 \mathrm{G}$ needle. For ionic hydrogel samples, a $2 \% \mathrm{w} / \mathrm{v}$ ultrapure alginate solution was prepared in DMEM and similarly mixed in a syringe with a $\mathrm{CaSO}_{4}$ slurry at a final concentration of $2 \% .50 \mathrm{uL}$ of the ionically crosslinked gel was also injected subcutaneously in the same mice. Both gel samples were retrieved along with the surrounding skin after 1 week, 1 month, and 2 months of injection and fixed overnight in 10\% neutral buffered formalin solution (Sigma-Aldrich). Samples were embedded in paraffin, sectioned, and stained with hematoxylin and eosin (H\&E) by the Harvard Rodent Histopathology Core.

\section{Results:}

3.1 Synthesis, characterization, and crosslinking of click alginate polymers 
To prepare click alginate polymers, norbornene or tetrazine groups were introduced to high molecular weight alginate biopolymers using conventional carbodiimide chemistry (Fig. 1-A). The degree of substitution of norbornene or tetrazine groups onto purified click alginate polymers was determined from ${ }^{1} \mathrm{H}$ NMR spectra (Fig. S-1). A 5\% degree of substitution of norbornene (Alg-N) or tetrazine (Alg-T) on alginate carboxyl groups was obtained using this method, and these batches of click alginate polymers were used for all subsequent experiments.

To form click alginate hydrogels, Alg-N and Alg-T polymer solutions were prepared separately and mixed together to gel. Upon mixing of the two click alginate polymers, a stable gel was formed via an inverse electron demand DielsAlder reaction between the two polymers, which releases nitrogen gas (Fig. 1-B). The nitrogen gas evolved from the crosslinking reaction does lead to the formation of a few small bubbles within the hydrogel. A stable gel was formed within 1 hour at $25^{\circ} \mathrm{C}$ (Fig. 2-A), though the gelation kinetics could be tuned by varying the temperature or initial degree of substitution of the click alginate polymers (data not shown). The gelation kinetics at $25{ }^{\circ} \mathrm{C}$ are favorable because it allows the user to easily achieve a well-mixed polymer formulation before gelation, a common challenge with other alginate hydrogel crosslinking methods.

\subsection{Compressive Young's modulus and swelling behavior}


The mechanical properties of the extracellular matrix have been shown to affect cell fate and function in 2D and 3D environments [34-37]. In order to tune mechanical properties over a wide range, click alginate polymers were mixed at different ratios of Alg-N and Alg-T (N:T ratio) for a given polymer concentration between 2 and $4 \% \mathrm{w} / \mathrm{v}$. These click alginate hydrogel samples were subjected to unconfined compression tests resulting in a compressive Young's modulus that predictably increased with increasing polymer concentration, and decreased as the ratio between the polymers deviated from the stoichiometrically balanced $\mathrm{N}: \mathrm{T}$ ratio of 1 (Fig. 2-B, Table S-1, Table S-2). The ability to tune the mechanical properties of the resulting gel over a large range by simply changing the ratio of the two polymers allows control over gel stiffness while keeping other parameters such as polymer concentration, and ligand density constant which may be useful for studies of mechanobiology.

The swelling ratio of hydrogel systems can affect mechanical properties, mass transport, and the presentation of ligands on the gel surface. To investigate how volumetric swelling would change at different polymer concentrations and $\mathrm{N}: \mathrm{T}$ ratios, click alginate hydrogels were made as previously described and allowed to swell for 24 hours at $37^{\circ} \mathrm{C}$. The swollen volume was measured and compared to the casted volume (Fig. 2-C). For a given polymer concentration, the volumetric swelling ratio increased as the N:T ratio deviated from 1, demonstrating an inverse relationship between mechanical properties and swelling ratio as expected. While the $\mathrm{N}: \mathrm{T}$ ratio has a significant effect on the 
swelling ratio, the polymer concentration does not have a significant effect, indicating that the swelling ratio of click alginate is dominated by crosslink density rather than polymer concentration (Table S-3).

\subsection{Post-gelation modification of click alginate hydrogels}

To explore if additional functionalities can be introduced to click alginate hydrogels after polymerization, we grafted thiol-containing molecules onto unreacted norbornenes in pre-formed click alginate hydrogels using a photoinitiated thiol-ene reaction (Fig. 3-A). Gels with N:T $=2$ were used to ensure unreacted norbornenes were available to react after the initial gelation. RGD peptide solutions at high $(2 \mathrm{mM})$ or low $(0.2 \mathrm{mM})$ concentration were reacted onto the surface of these click alginate hydrogels and then gels were seeded with $\mathrm{NIH}$ 3T3 fibroblasts expressing a cytosolic fluorescent marker (EGFP). 3T3 cells readily adhered and spread on gels modified with RGD, while very few cells were able to attach or elongate on control gels with no RGD (Fig. 3-B). Cells on click alginate hydrogels presenting RGD were able to form branched interconnected networks, with a significant RGD density-dependent 2-3 fold increase in surface coverage over the 3 day culture, while unmodified click alginate gels were observed to be non-cell-adhesive and showed a decrease in surface coverage by cells over time (Fig. 3-C). After 3 days in culture, cells also showed an increase in spreading and actin stress fiber formation with higher RGD concentration (Fig. 3- 
D). Additionally, the high viability of cells after 3 days of culture demonstrated the cytocompatibility of the click alginate hydrogels for 2D cell culture (Fig. 3-E).

\subsection{Cell encapsulation in click alginate hydrogels}

In order to demonstrate the utility of click alginate hydrogels for cell encapsulation, cell viability and metabolic activity of cells encapsulated in click alginate hydrogels were investigated over a 3 day culture period; ionically crosslinked hydrogels were used for comparison in these studies. Representative images of encapsulated cells stained with ethidium homodimer-1 show minimal cell death in both click and ionically crosslinked gels 4 hours and 3 days after encapsulation (Fig. 4-A). Quantification revealed that click alginate hydrogels resulted in significantly higher viability of encapsulated 3 T3 cells both immediately after encapsulation ( $93 \pm 1 \%$ vs. $87 \pm 2 \%$ ) and after 3 days of culture ( $84 \pm 2 \%$ vs. $79 \pm 4 \%$ ) (Fig. $4-B)$. It should be noted that a loss in measured cell viability may occur during the cell retrieval process by enzymatic digestion of the hydrogels. The overall metabolic activity of the cells encapsulated in the different hydrogels was also analyzed, and noted to increase over the 3 day culture period for both hydrogel crosslinking chemistries (Fig. 4-C).

\subsection{In vivo injection}

The inflammatory response to the injection of click alginate hydrogels in vivo was investigated next. Click crosslinked and ionically crosslinked alginate 
hydrogels were injected subcutaneously and retrieved after 1 week, 1 month, and 2 months. The gelation kinetics of click alginate hydrogels allows them to be mixed and readily injected, in a similar manner to ionically crosslinked hydrogels. A thin fibrous capsule was found to surround both types of gels 1 week after injection. H\&E staining revealed a very thin capsule of collagen and fibroblasts surrounding the material throughout the duration of the study with minimal inflammation (Fig. 5). At 1 month, the ionically crosslinked gels were seen to lose structural integrity and allowed for infiltration of fibroblasts and immune cells into the gel, while the click crosslinked samples showed no evidence of breakdown nor cell infiltration into the material for up to 2 months (see Fig. S-2), and maintained a thin layer of fibroblasts surrounding the gel.

\section{Discussion:}

Our results show that alginate polymers can be modified with norbornene and tetrazine to create alginate hydrogels with a wide-range of mechanical properties without the input of external energy, crosslinkers, or catalysts. While recent work has used similar click chemistry for localized drug delivery, this work presents the first use of the tetrazine-norbornene click reaction to covalently crosslink polysaccharides into hydrogels $[29,38]$. Crosslinking of alginate by different methods has been extensively explored to make covalently crosslinked hydrogels that are mechanically robust, but these chemistries lack the cytocompatibility inherent in the bioorthogonal click reaction reported here $[19,21,39]$. The 
simplicity of this crosslinking modality provides the opportunity to control the mechanical properties of the click alginate hydrogel by adjusting the ratio of the polymers, rather than changing the total concentration of polymers in the system. This could potentially allow for the decoupling of material variables such as gel architecture, stiffness, and ligand density in further applications of click alginate hydrogels.

Click crosslinked alginate hydrogels were used to form a cytocompatible 2D cell culture substrate that can be modified to display cell adhesion peptides at varying concentrations. Alginate hydrogels must display cell adhesive ligands in order for mammalian cells to attach, spread, and proliferate on the surface of the hydrogel. Without ligands such as RGD presented from the hydrogel surface, few cells will attach, and those that do will retain a spherical morphology and undergo apoptosis [21]. Unfortunately, the carbodiimide chemical reaction most commonly used to attach RGD peptides to the backbone of alginate is slow and requires lengthy purification and lyophillization time [40]. In this work, photoinitated thiolene chemistry between norbornene and cysteine-bearing RGD peptides was employed to rapidly modify click alginate hydrogels to present adhesion ligands on the surface of the gel. This thiol-ene reaction is a powerful light-mediated click reaction that is simple, reproducible, fast, and highly efficient - achieving conversions nearing completion in aqueous media [41]. Although we did not investigate the thiol-ene reaction conversion as a function of hydrogel depth specifically, several recent papers have reported the ability to functionalize the 
interiors of hydrogels using this method $[28,30,42,43]$. When click alginate hydrogels were modified with RGD peptides using this strategy, fibroblasts seeded on the gels responded with increased attachment and spreading as RGD density was raised, over a 3 day culture period. In addition to the simple and rapid coupling reaction, the thiol-ene based strategy for modifying alginate hydrogels also presents a straightforward method to change the ligand density on hydrogels of otherwise equal composition. Altogether, these data demonstrate the flexibility of click alginate hydrogels for culturing cells in 2D and allowing independent control over the presentation of bioactive ligands on the gel surface.

Furthermore, click crosslinked alginates can be used in vitro to encapsulate cells in 3D with high viability, providing a covalent alternative to conventional ionically crosslinked alginate hydrogels. A variety of cell types have been encapsulated in ionically crosslinked RGD modified alginates with high viability in vitro $[11,35,44-46]$. However, encapsulation of cells in covalently crosslinked RGD modified alginates is limited by the potential incompatibility of the available crosslinking chemistries $[47,48]$. The data shown here establishes the ability to encapsulate fibroblasts in covalently crosslinked RGD modified click alginate hydrogels while maintaining cell viability at a high level. The aforementioned ability to independently tune the microenvironment mechanical properties and adhesion ligand density can be exploited with the click crosslinked 3D cell culture system in the future to probe cell responses to a variety of stimuli in vitro. 
In vivo testing showed that click alginate hydrogels can crosslink in situ, provoke minimal inflammatory response, and resist fragmentation and cell infiltration when injected subcutaneously. Histology revealed minimal acute inflammation in the tissue surrounding the injected gel in both click crosslinked and ionically crosslinked alginate. As is typical with many biomaterials, a small fibrotic capsule was formed around the hydrogel periphery in both cases [49]. When compared to ionically crosslinked alginate, click alginate hydrogels demonstrate superior long-term structural integrity. Ionically crosslinked samples fragmented significantly after 1 month in vivo, resulting in cell infiltration, whereas the click alginate hydrogels remained intact during the 2 month study and were highly resistant to cell infiltration. In tissue engineering applications where cell trafficking within the hydrogel is desirable, click alginate hydrogels could be processed using existing techniques to introduce microscale porosity to the hydrogels $[50,51]$. Alternatively, click alginate polymers could be crosslinked using tetrazine or norbornene-modified matrix metalloproteinase-degradable peptide sequences to allow cell-mediated degradation [29,52]. The use of partially oxidized alginate polymers would also allow degradation of the hydrogel over controlled time scales for in vivo tissue engineering applications [20,53]. The tissue compatibility and stability of click alginate hydrogels could make it particularly useful for applications where isolation from host immune cell infiltration is required $[54,55]$. 


\section{Conclusions:}

Click alginate polymers are synthetically accessible and can be crosslinked in biological media at physiological $\mathrm{pH}$ to create tunable hydrogels with a wide range of mechanical properties. The rapid, bioorthogonal, and cytocompatible click crosslinking reaction makes click alginate hydrogels favorable for cell engineering applications. Click alginate hydrogels can be quickly modified to be cell adhesive and used for 2D or 3D cell culture. Additionally, click alginates have a minimal inflammatory response and high stability in vivo, making them attractive materials to use for long-term cell encapsulation and biomaterialsbased tissue engineering applications.

\section{Acknowledgements:}

This work was supported by the Army Research Office (W911NF-13-1-0242) and the NIH (R01 DE013349). This work was performed in part at the MGH Center for Systems Biology. The authors would like to acknowledge the help of Olivier Kister, Kaixiang Lin, and Chris Johnson for material synthesis and troubleshooting. The authors would also like to thank Dr. Luo Gu, Dr. Ovijit Chaudhuri, Daniel Rubin, Alexander Cheung, Dr. Catia Verbeke, Zsofia Botiyanski, Ajay Parmar, and Max Darnell for scientific discussions.

\section{Appendix}

\section{Supplementary data}




\section{References:}

[1] Langer R, Vacanti JP. Tissue engineering. Science 1993;260:920-6.

[2] Ratner BD, Bryant SJ. Biomaterials: where we have been and where we are going. Annu Rev Biomed Eng 2004.

[3] Drury JL, Mooney DJ. Hydrogels for tissue engineering: scaffold design variables and applications. Biomaterials 2003;24:4337-51.

[4] Kearney CJ, Mooney DJ. Macroscale delivery systems for molecular and cellular payloads. Nature Materials 2013;12:1004-17.

[5] Conway A, Schaffer DV. Biomaterial microenvironments to support the generation of new neurons in the adult brain. Stem Cells 2014;32:1220-9.

[6] Huebsch N, Kearney CJ, Zhao X, Kim J, Cezar CA, Suo Z, et al. Ultrasound-triggered disruption and self-healing of reversibly cross-linked hydrogels for drug delivery and enhanced chemotherapy. Proceedings of the National Academy of Sciences 2014;111:9762-7.

[7] Hori Y, Winans AM, Huang CC, Horrigan EM, Irvine DJ. Injectable dendritic cell-carrying alginate gels for immunization and immunotherapy. Biomaterials 2008;29:3671-82.

[8] Martinsen A, Skjåk-Braek G, Smidsrød O. Alginate as immobilization material: I. Correlation between chemical and physical properties of alginate gel beads. Biotechnol Bioeng 1989;33:79-89.

[9] Augst AD, Kong HJ, Mooney DJ. Alginate Hydrogels as Biomaterials. Macromol Biosci 2006;6:623-33.

[10] Freeman I, Kedem A, Cohen S. The effect of sulfation of alginate hydrogels on the specific binding and controlled release of heparinbinding proteins. Biomaterials 2008;29:3260-8.

[11] Madl CM, Mehta M, Duda GN, Heilshorn SC, Mooney DJ. Presentation of BMP-2 mimicking peptides in 3D hydrogels directs cell fate commitment in osteoblasts and mesenchymal stem cells. Biomacromolecules 2014;15:445-55.

[12] Boontheekul T, Kong HJ, Mooney DJ. Controlling alginate gel degradation utilizing partial oxidation and bimodal molecular weight distribution. Biomaterials 2005;26:2455-65.

[13] Coviello T, Matricardi P, Marianecci C, Alhaique F. Polysaccharide hydrogels for modified release formulations. J Control Release 2007;119:5-24.

[14] Shoichet MS, Li RH, White ML, Winn SR. Stability of hydrogels used in cell encapsulation: An in vitro comparison of alginate and agarose. Biotechnol Bioeng 1996;50:374-81.

[15] Kuo CK, Ma PX. lonically crosslinked alginate hydrogels as scaffolds for tissue engineering: Part 1. Structure, gelation rate and mechanical properties. Biomaterials 2001;22:511-21. 
[16] Chan G, Mooney DJ. $\mathrm{Ca}(2+)$ released from calcium alginate gels can promote inflammatory responses in vitro and in vivo. Acta Biomaterialia 2013;9:9281-91.

[17] Rowley JA, Madlambayan G, Mooney DJ. Alginate hydrogels as synthetic extracellular matrix materials. Biomaterials 1999;20:45-53.

[18] Seliktar D. Designing Cell-Compatible Hydrogels for Biomedical Applications. Science 2012;336:1124-8.

[19] Eiselt P, Lee KY, Mooney DJ. Rigidity of Two-Component Hydrogels Prepared from Alginate and Poly(ethylene glycol)-Diamines. Macromolecules 1999;32:5561-6.

[20] Bouhadir KH, Hausman DS, Mooney DJ. Synthesis of cross-linked poly (aldehyde guluronate) hydrogels. Polymer 1999;40:3575-84.

[21] Jeon O, Bouhadir KH, Mansour JM, Alsberg E. Photocrosslinked alginate hydrogels with tunable biodegradation rates and mechanical properties. Biomaterials 2009;30:2724-34.

[22] Lee KY, Rowley JA, Eiselt P, Moy EM, Bouhadir KH, Mooney DJ. Controlling mechanical and swelling properties of alginate hydrogels independently by cross-linker type and cross-linking density. Macromolecules 2000.

[23] Tibbitt MW, Anseth KS. Dynamic microenvironments: the fourth dimension. Science Translational Medicine 2012;4:160ps24-4.

[24] Jewett JC, Bertozzi CR. Cu-free click cycloaddition reactions in chemical biology. Chem Soc Rev 2010;39:1272-9.

[25] Devaraj NK, Weissleder R, Hilderbrand SA. Tetrazine-Based Cycloadditions: Application to Pretargeted Live Cell Imaging. Bioconjugate Chem 2008;19:2297-9.

[26] DeForest CA, Anseth KS. Cytocompatible click-based hydrogels with dynamically tunable properties through orthogonal photoconjugation and photocleavage reactions. Nat Chem 2011;3:925-31.

[27] DeForest CA, Polizzotti BD, Anseth KS. Sequential click reactions for synthesizing and patterning three-dimensional cell microenvironments. Nature Materials 2009;8:659-64.

[28] Fairbanks BD, Schwartz MP, Halevi AE, Nuttelman CR, Bowman CN, Anseth KS. A Versatile Synthetic Extracellular Matrix Mimic via ThiolNorbornene Photopolymerization. Adv Mater 2009;21:5005-10.

[29] Alge DL, Azagarsamy MA, Donohue DF, Anseth KS. Synthetically Tractable Click Hydrogels for Three-Dimensional Cell Culture Formed Using Tetrazine-Norbornene Chemistry. Biomacromolecules 2013;14:949-53.

[30] Aimetti AA, Machen AJ, Anseth KS. Poly(ethylene glycol) hydrogels formed by thiol-ene photopolymerization for enzyme-responsive protein delivery. Biomaterials 2009;30:6048-54.

[31] Shih H, Lin C-C. Cross-Linking and Degradation of Step-Growth Hydrogels Formed by Thiol-Ene Photoclick Chemistry. 
Biomacromolecules 2012;13:2003-12.

[32] Karver MR, Weissleder R, Hilderbrand SA. Synthesis and evaluation of a series of 1,2,4,5-tetrazines for bioorthogonal conjugation. Bioconjugate Chem $2011 ; 22: 2263-70$.

[33] Pfeifer A, Ikawa M, Dayn Y, Verma IM. Transgenesis by lentiviral vectors: lack of gene silencing in mammalian embryonic stem cells and preimplantation embryos. Proc Natl Acad Sci USa 2002;99:2140-5.

[34] Engler AJ, Sen S, Sweeney HL, Discher DE. Matrix elasticity directs stem cell lineage specification. Cell 2006;126:677-89.

[35] Huebsch N, Arany PR, Mao AS, Shvartsman D, Ali OA, Bencherif SA, et al. Harnessing traction-mediated manipulation of the cell/matrix interface to control stem-cell fate. Nature Materials 2010;9:518-26.

[36] Khetan S, Guvendiren M, Legant WR, Cohen DM, Chen CS, Burdick JA. Degradation-mediated cellular traction directsstem cell fate in covalently crosslinkedthree-dimensional hydrogels. Nature Materials 2013;12:1-8.

[37] Chaudhuri O, Koshy ST, Branco da Cunha C, Shin J-W, Verbeke CS, Allison $\mathrm{KH}$, et al. Extracellular matrix stiffness and composition jointly regulate the induction of malignant phenotypes in mammary epithelium. Nature Materials 2014;13:970-8.

[38] Mejía Oneto JM, Gupta M, Leach JK, Lee M, Sutcliffe JL. Implantable biomaterial based on click chemistry for targeting small molecules. Acta Biomaterialia 2014;10:5099-105.

[39] Lee KY, Bouhadir KH, Mooney DJ. Controlled degradation of hydrogels using multi-functional cross-linking molecules. Biomaterials 2004;25:2461-6.

[40] Rowley JA, Mooney DJ. Alginate type and RGD density control myoblast phenotype. J Biomed Mater Res 2002;60:217-23.

[41] Hoyle CE, Bowman CN. Thiol-Ene Click Chemistry. Angew Chem Int Ed 2010;49:1540-73.

[42] Gramlich WM, Kim IL, Burdick JA. Synthesis and orthogonal photopatterning of hyaluronic acid hydrogels with thiol-norbornene chemistry. Biomaterials 2013;34:9803-11.

[43] Mũnoz Z, Shih H, Lin C-C. Gelatin hydrogels formed by orthogonal thiolnorbornene photochemistry for cell encapsulation. Biomater Sci 2014;2:1063-72.

[44] Fonseca KB, Gomes DB, Lee K, Santos SG, Sousa A, Silva EA, et al. Injectable MMP-sensitive alginate hydrogels as hMSC delivery systems. Biomacromolecules 2014;15:380-90.

[45] Nakaoka R, Hirano Y, Mooney DJ, Tsuchiya T, Matsuoka A. Study on the potential of RGD- and PHSRN-modified alginates as artificial extracellular matrices for engineering bone. J Artif Organs 2013;16:284-93.

[46] Kreeger PK, Deck JW, Woodruff TK, Shea LD. The in vitro regulation of ovarian follicle development using alginate-extracellular matrix gels. Biomaterials 2006;27:714-23. 
[47] Lee KY, Alsberg E, Mooney DJ. Degradable and injectable poly(aldehyde guluronate) hydrogels for bone tissue engineering. J Biomed Mater Res 2001;56:228-33.

[48] Jeon O, Alsberg E. Photofunctionalization of alginate hydrogels to promote adhesion and proliferation of human mesenchymal stem cells. Tissue Eng Part A 2013;19:1424-32.

[49] Mikos A, Mclntire L, Anderson J, Babensee J. Host response to tissue engineered devices. Advanced Drug Delivery Reviews 1998;33:111-39.

[50] Annabi N, Nichol JW, Zhong X, Ji C, Koshy S, Khademhosseini A, et al. Controlling the porosity and microarchitecture of hydrogels for tissue engineering. Tissue Engineering Part B: Reviews 2010;16:371-83.

[51] Koshy ST, Ferrante TC, Lewin SA, Mooney DJ. Injectable, porous, and cell-responsive gelatin cryogels. Biomaterials 2014;35:2477-87.

[52] Lutolf MP, Raeber GP, Zisch AH, Tirelli N, Hubbell JA. Cell-Responsive Synthetic Hydrogels. Adv Mater Weinheim 2003;15:888-92.

[53] Lee KY, Bouhadir KH, Mooney DJ. Degradation behavior of covalently cross-linked poly (aldehyde guluronate) hydrogels. Macromolecules 2000.

[54] Jacobs-Tulleneers-Thevissen D, Chintinne M, Ling Z, Gillard P, Schoonjans L, Delvaux G, et al. Sustained function of alginateencapsulated human islet cell implants in the peritoneal cavity of mice leading to a pilot study in a type 1 diabetic patient. Diabetologia 2013;56:1605-14.

[55] Ma M, Chiu A, Sahay G, Doloff JC, Dholakia N, Thakrar R, et al. Coreshell hydrogel microcapsules for improved islets encapsulation. Adv Healthc Mater 2013;2:667-72. 


\section{Figure Legends:}

Fig. 1. Fabrication of click alginate hydrogels. Schematic of click alginate polymer synthesis. Aqueous carbodiimide chemistry is used to modify alginate backbone carboxylic acids with tetrazine or norbornene, resulting in Alg-T or Alg-N polymers respectively (A). Alg-T and Alg-N polymers are mixed together to create a covalently crosslinked click alginate hydrogel network, with the loss of $\mathrm{N}_{2}$ (B).

Fig. 2. Click alginate hydrogel mechanical properties. Representative in situ dynamic rheometry plot at $25^{\circ} \mathrm{C}$ for $3 \% \mathrm{w} / \mathrm{v}$ click alginate at $\mathrm{N}: \mathrm{T}=1$, demonstrating modulus evolution with time (A). Compressive Young's modulus (B) and volumetric swelling ratios (C) for $2 \%, 3 \%$ and $4 \% \mathrm{w} / \mathrm{v}$ click alginate hydrogels at varying N:T ratio. Values represent mean and standard deviation ( $n$ $=4)$.

Fig. 3. Cell adhesion, spreading, and proliferation on click alginate hydrogels modified with RGD peptides after synthesis. Schematic of CGGGGRGDSP peptide coupling reaction onto click alginate hydrogel surface using photoinitiated thiol-ene chemistry (A). Representative images of 3T3 fibroblast adhesion, spreading, and proliferation on click alginate hydrogels with varying RGD peptide density (scale bar $=200 \mu \mathrm{m})(\mathrm{B})$, and quantification (Two-Way ANOVA with Turkey's post-hoc test, ${ }^{*} p<0.05,{ }^{* * * *} p<0.0001$ relative to No RGD control; 
Values represent mean and standard deviation, $n=4-7$ ) by endogenous EGFP expression (green) over 3 days (C). Phalloidin (red) and Hoescht 33342 (blue) staining of F-actin filaments and nuclei at 3 days for cells adherent to RGD modified click alginate hydrogels (scale bar $=100 \mu \mathrm{m})(\mathrm{D})$. Representative fluorescent images of EGFP (green) 3T3 cells cultured on click alginate hydrogels with varying ligand density for 3 days and stained with ethidium homodimer-1 (red) $($ scale bar $=100 \mu \mathrm{m})(\mathrm{E})$. The High, Low, and No RGD conditions refer to the $2 \mathrm{mM}, 0.2 \mathrm{mM}$, and $0 \mathrm{mM}$ peptide solutions used to modify the click alginate hydrogel surface.

Fig. 4. Cell encapsulation in click crosslinked and ionically crosslinked alginate hydrogels. 3T3 fibroblasts were encapsulated in $2 \%$ w/v click crosslinked (N:T = 1) and ionically crosslinked alginate hydrogels and stained with ethidium homodimer-1 (red) for dead cells at 4 hours and 3 days post encapsulation (scale bar $=100 \mu \mathrm{m})(\mathrm{A})$. Quantitative analysis of cell viability (Two-Way ANOVA with Sidak's post-hoc test, ${ }^{\star \star} p<0.01,{ }^{\star * *} p<0.001$; Values represent mean and standard deviation, $n=4$ ) and overall metabolic activity as measured by reduction of AlamarBlue over time in culture $(n=6)(\mathrm{B})$.

Fig. 5. Tissue response following subcutaneous injection of click and ionically crosslinked hydrogels in vivo. Representative hematoxylin and eosin (H\&E) stain of tissue sections at 1 week, 1 month, and 2 month following injection into 
BALB/cJ mice (scale bar $=150 \mu \mathrm{m}$ ). Images focus on the gel-tissue interface, with dashed lines indicating the border between the hydrogel and the surrounding tissue. Asterisks indicate the location of the click alginate hydrogel, which separates from the tissue during histological analysis with no cell infiltration.

\section{Supplementary Information}

\section{Supplementary Methods:}

\section{${ }^{1} H N M R$}

Alg- $\mathrm{N}, \mathrm{Alg}-\mathrm{T}$, and unmodified alginate polymers were dissolved in deuterium oxide (Sigma-Aldrich) at $1.5 \% \mathrm{w} / \mathrm{v} .{ }^{1} \mathrm{H}$ NMR spectra were obtained on a $400 \mathrm{MHz}$ NMR spectrometer (Varian). The degree of substitution was calculated by comparing the integral of the alginate backbone proton peaks at $\delta 5.0$ and $\delta 4.5$ with either the alkene proton peaks of norbornene at $\delta 6.2-5.9(\mathrm{~m}, 2 \mathrm{H})$ or the aromatic proton peak of tetrazine at $\delta 10.4(\mathrm{~s}, 1 \mathrm{H})$.

\section{Supplementary Figure Legends:}

Fig. S-1. ${ }^{1} \mathrm{H}$ NMR spectra of unmodified alginate and click alginate polymers. Blue box highlights the appearance of alkene protons in Alg- $\mathrm{N}$ spectra and red box highlights aromatic protons in Alg-T spectra after coupling reaction of norbornene and tetrazine onto alginate. 
Fig. S-2. H\&E of click and ionically crossslinked alginate hydrogel. Images focus on interior of the hydrogel at 2 months following subcutaneous injection in vivo (scale bar $=200 \mu \mathrm{m})$.

Table S-1. Young's modulus statistical differences between polymer concentration at each N:T ratio. Values calculated using Two-Way ANOVA with Turkey's post-hoc test, ${ }^{*} p<0.05,{ }^{* \star} p<0.01,{ }^{* \star *} p<0.001,{ }^{* \star * *} p<0.0001, x=$ not significantly different.

Table S-2. Young's modulus statistical differences between N:T ratio at each polymer concentration. Values calculated using Two-Way ANOVA with Turkey's post-hoc test, ${ }^{*} p<0.05,{ }^{* *} p<0.01,{ }^{* \star *} p<0.001,{ }^{* \star *} p<0.0001, x=$ not significantly different.

Table S-3. Swelling ratio statistical differences between N:T ratio at each polymer concentration. Values calculated using Two-Way ANOVA with Turkey's post-hoc test, ${ }^{*} p<0.05,{ }^{* *} p<0.01,{ }^{* * *} p<0.001,{ }^{* * * *} p<0.0001, x=$ not significantly different. 